\title{
A prioritisation system for elective coronary angiography
}

\author{
D P de Bono, B Ravilious, I El-Zoubi, T Dyer, Y Podinovskaya
}

\begin{abstract}
Objective-To devise a clinical prioritisation system for rationing access to a cardiac catheter waiting list and to describe its performance at predicting angiographic findings and selecting patients for angioplasty or coronary artery bypass graft surgery.

Setting-Tertiary level cardiology centre. Methods-(1) 665 consecutive patients on an elective waiting list for coronary angiography were scored using a system derived from established clinical criteria for selecting patients for coronary surgery (New Zealand/Duke). The scores were compared with clinical outcome (referral for surgery, angioplasty, or medical management). (2) In a subset of 125 patients, scores derived from clinical criteria and exercise testing were compared with findings on coronary angiography. (3) Multivariate analysis was used in a new group of 178 patients to identify factors that would be better predictors of the angiographic score. (4) A new scoring system was devised based partly on the results of the multivariate analysis. It was applied to a new test group of 100 patients using clinical outcome and angiographic score as outcome measures.
\end{abstract}

Results-(1) Using the established clinical score, similar proportions of patients were referred after angiography for medical management, angioplasty, or coronary bypass grafting, irrespective of their original score. The exceptions were patients with a score $<20$, who were more likely to continue medical management. (2) There was poor correlation $(r=0.05)$ between the clinical score and the subsequent angiographic score. (3) Multivari-

Accepted for publication 13 January 1998

Table 1 Scoring factors in non-invasive prioritisation scheme: first version

\begin{tabular}{llr}
\hline Heading & Details & Score \\
\hline Angina & Class I: angina on strenuous exertion & 1 \\
& Class II: angina on walking/climbing stairs rapidly & 2 \\
& Class III: angina walking one or two level blocks & 8 \\
Exercise test & Class IV: unstable angina/rest pain & 18 \\
& Bruce stage IV or negative at stage III & 0 \\
& Stage III positive & 8 \\
& Stage II positive & 12 \\
No of antianginal drugs & Could not exercise & 22 \\
& None (excluding GTN) & 10 \\
& One & 0 \\
& Two & 5 \\
Previous MI & Three & 10 \\
Previous unstable angina & More than three & 15 \\
& & 20 \\
& & 10 \\
& & 10
\end{tabular}

Possible maximum score is 80 , allowing a further 20 points for social factors.

Fields in italics were not included in original criteria.

GTN, glyceryl trinitrate; MI, myocardial infarction. ate analysis identified age, male sex, previous myocardial infarction, high cholesterol, and diabetes as independent predictors of coronary score. (4) The modified scoring system, incorporating the predictors identified by multivariate analysis, performed better than the original scoring system in predicting coronary score when both were tested, but some patients had severe disease despite a low score.

Conclusions-Patients can be ranked using clinical and non-invasive criteria, and a rationing system implemented on this basis. With prioritisation by noninvasive criteria, the risk of missing serious coronary disease in patients with relatively mild symptoms must be accepted; this risk becomes greater the more stringently rationing is applied. (Heart 1998;79:448-453)

Keywords: prioritisation; coronary angiography

Coronary arteriography is now a routine investigation in patients with angina or other forms of suspected coronary artery disease. ${ }^{1}$ Access to coronary arteriography in Great Britain has traditionally been restricted by financial limitations, and the problem is further exacerbated by regional variations in referral rate. ${ }^{23} \mathrm{Re}-$ peated attempts have been made to provide a rational basis for planning the necessary number of coronary arteriograms for given populations, ${ }^{4-6}$ but these were hampered by incomplete epidemiological data, and have been overtaken by developments, in particular the extension of effective intervention to older patients.

For several years providers of cardiac services have been aware of increasing tension between the demand for cardiac investigation and intervention, particularly for coronary disease, and the level of funding available to provide service. The problem has been complicated by the NHS reforms of 1991 with the introduction of an internal market and multiple levels of funding, and further affected by the patient's charter requirement that all procedures should take place within one year of a patient being placed on a waiting list. Implicit rationing has always been a feature of NHS provision, but we became convinced that a more formal, equitable, and transparent system was necessary.

The issues to be addressed in designing such a rationing system are complex. It would be helpful to have a system that will select for angiography the patients most likely to require 
Table 2 Angiographic scoring factors (adapted from reference 8)

\begin{tabular}{lc}
\hline Extent of coronary disease & Score \\
\hline No stenosis $>50 \%$ & 0 \\
1 vessel disease $50-74 \%$ & 8 \\
$>$ 1 vessel disease $(50-74 \%)$ & 9 \\
1 vessel disease $(75-89 \%)$ & 9 \\
1 vessel disease $(>90 \%)$ & 14 \\
2 vessel disease $(50-89 \%)$ & 15 \\
2 vessel disease $(>90 \%)$ not involving LAD & 15 \\
1 vessel disease $(>90 \%)$ involving proximal LAD & 19 \\
2 vessel disease $(>90 \%)$ involving LAD & 19 \\
3 vessel disease & 19 \\
3 vessel disease $(>90 \%)$ in at least one & 19 \\
3 vessel disease with $75-89 \%$ stenosis involving & \\
proximal LAD & 19 \\
3 vessel disease with $>90 \%$ stenosis involving & 27 \\
proximal LAD & 27 \\
Left main $(50 \%)$ & 32 \\
Left main $(75-89 \%)$ & 36 \\
Left main (>90\%) & \\
\hline
\end{tabular}

LAD, left anterior descending coronary artery.

angioplasty or coronary bypass grafting. However, there is also a subgroup of patients with severe symptoms but normal coronary arteriograms in whom it can be shown that coronary angiography is very cost-effective. In deciding who requires intervention should one's main aim be relief of symptoms or improvement in survival? As Gray and Hampton have pointed out, ${ }^{7}$ these two aims lead to different selection criteria and indeed to different selection strategies. If the aim is to produce maximum relief of symptoms then selection should emphasise symptomatic criteria and the aim will be to perform the maximum number of interventions (angioplasty or bypass grafting) for the minimum number of diagnostic procedures. Conversely if the aim is to select patients whose lives will be prolonged the best strategy may be to perform a large number of diagnostic procedures but to limit bypass grafting to those patience with severe coronary disease (and omit angioplasty altogether).

One of the few attempts to reconcile the two points of view has been the New Zealand

Table 3 Expanded clinical information collected in patients undergoing angiography

\begin{tabular}{lcl}
\hline & $\begin{array}{l}\text { Patients in multivariate } \\
\text { analysis } \%(n=178)\end{array}$ & $\begin{array}{l}\text { Patients used to test } \\
\text { modified score } \% \\
(n=100)\end{array}$ \\
Patient characteristics & 74 & 73 \\
Male & 26 & 27 \\
Female & 41 & 36 \\
Age (years) & 52 & 58 \\
$\quad<59$ & 7 & 6 \\
$60-74$ & 12 & 7 \\
$>75$ & 13 & 16 \\
Smoker & 18 & 26 \\
Diabetes & 28 & 26 \\
Hypertension & 95 & 95 \\
Cholesterol > 6.5 mmol/1 & & \\
Symptomatic angina & 7 & 3 \\
Antianginal drugs & 27 & $15^{\star}$ \\
None & 43 & 44 \\
1 drug & 22 & $38^{\star}$ \\
2 drugs & & 20 \\
$>2$ drugs & 19 & 69 \\
Exercise test & 61 & 11 \\
$\quad$ Not done & 20 & $57^{\star}$ \\
Positive & 34 & 22 \\
$\quad$ Negative/equivocal & 17 & 53 \\
Previous myocardial infarct & & 25 \\
Previous angioplasty or bypass graft & 42 & 22 \\
Outcome & 33 & \\
Medical management & 25 & \\
Refer for angioplasty & & \\
Refer for bypass graft & & \\
\hline
\end{tabular}

^Significant difference at $5 \%$ level.

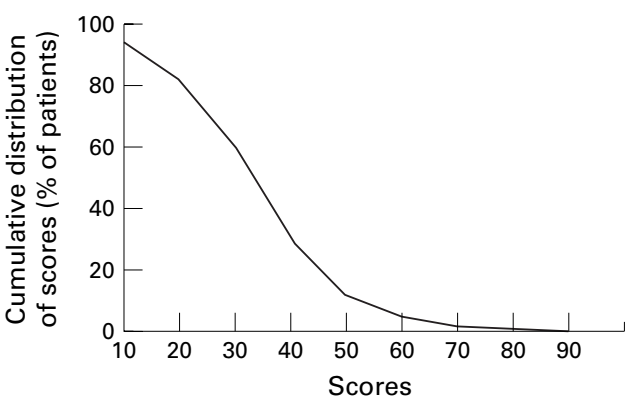

Figure 1 Cumulative frequency curve for non-invasive scores applied to 665 catheter waiting list patients.

priority criteria project. $^{8}$ This part evidence based, part consensus based scheme uses clinical, angiographic, and social criteria to construct a numerical priority scale. This scale is then used as an instrument for rationing access to coronary bypass graft surgery. Inspired by this, we set out to investigate whether a similar concept could be used to prioritise patients for diagnostic coronary angiography.

We describe the project in two phases. In the first phase we devised a prioritisation scheme based on clinical data, applied it retrospectively to patients undergoing angiography, and measured its performance in terms of predicting coronary angiographic findings and the selection of patients for angioplasty, bypass grafting, or medical management. We found that neither symptom severity nor exercise testing were good predictors of the extent of disease found at coronary angiography. A second phase of the project then involved the use of multivariate analysis to identify features that would be independent predictors of coronary score. We incorporated these into a new scoring system, which we tested in a further 100 patients.

\section{Methods}

Glenfield hospital is a tertiary cardiac centre serving a population of approximately 1.8 million. About 2500 cardiac catheter studies are performed each year, of which some two thirds are elective procedures. The majority of these cases are initially seen in the outpatient clinic, where the decision to proceed to a catheter study is usually made. As part of a prospective contract monitoring exercise begun in June 1996, clinical data are collected at the time the patient is placed on the waiting list, using a standard data collection form.

INITIAL MAPPING OF WAITING LIST PATIENTS TO A CLINICAL SCORING SYSTEM

A clinical scoring system based on that used in the New Zealand priority criteria project was drawn up. The data used and the weighting attached to the different findings are shown in table 1 . Weightings for angina severity and for exercise test performance were adapted directly from the New Zealand/Duke criteria. ${ }^{89}$ Because we were dealing with an outpatient population, the weighting used for Canadian Cardiovascular Society class 4 angina was that given for class $4 \mathrm{~A}$. We gave additional pointsfor previous myocardial infarction, unstable angina, and the number of antianginal drugs- 


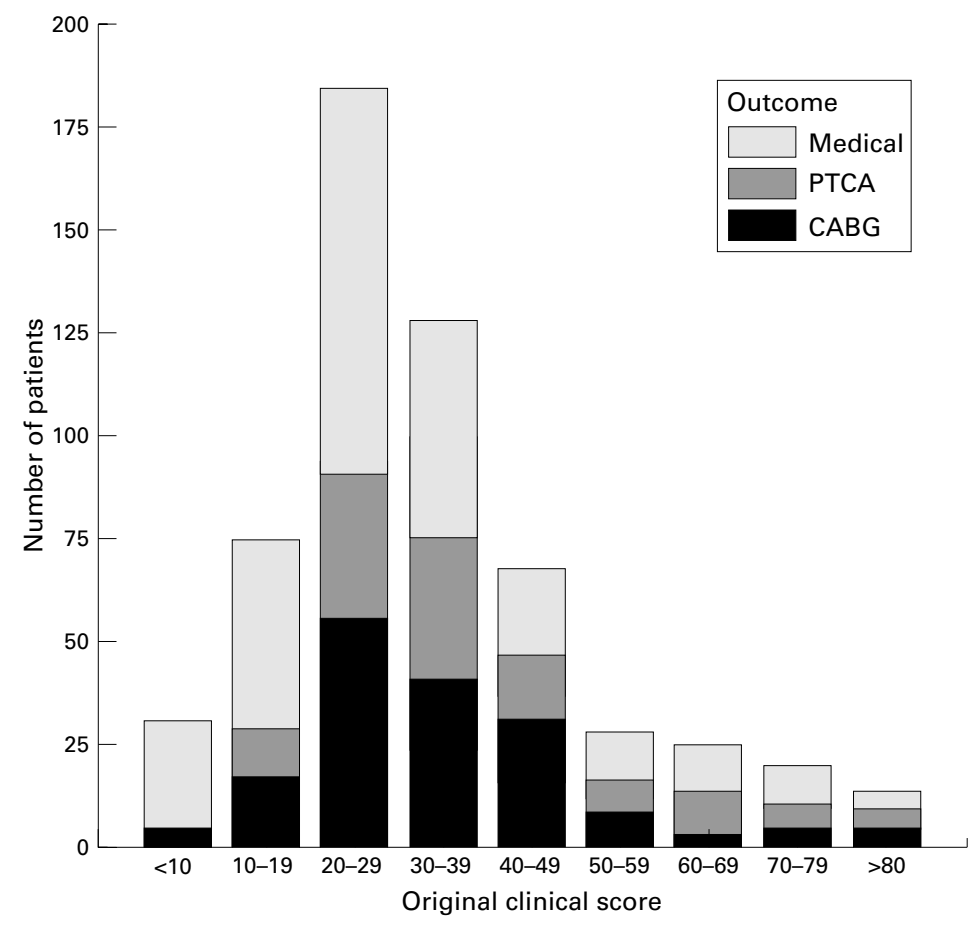

Figure 2 Relation between original clinical score and postangiography management decision for 572 patients for whom outcome data were available.

which were not specified in the original published criteria. These weightings were reached by consensus among the six cardiologists in our unit. We omitted data about the extent of coronary artery disease, since these were obviously not available before cardiac catheterisation. We also omitted data about the social impact of the symptoms, since these were not specified in our data collection form, designed before publication of the New Zealand project. Scores for each of the clinical criteria were summed and multiplied by a scaling factor of 1.2 to convert the scores to a scale with a maximum of 100 . Clinical outcome data were obtained from the case records and checked against angioplasty and surgical waiting lists. For the purposes of this study we used only the decisions made at the time of the coronary angiogram or the first postangiogram outpatient visit.

\section{COMPARISON OF CLINICAL SCORE WITH}

ANGIOGRAPHIC DATA

Comparison was made between the noninvasive score and angiographic data in a subset comprising the 125 consecutive patients who had undergone angiography by March 1997. Angiographic data were scored as shown in table 2 , on the basis of the New Zealand priority criteria project. Angiographic data were abstracted from case records. Where the report

Table 4 Cumulative adjusted $R^{2}$, coefficients, and standard error for multivariate predictors of coronary artery score

\begin{tabular}{lcll}
\hline Variable & Adjusted $R^{2}$ & Coefficients & Standard error \\
\hline Previous MI & 8.4 & 4.26 & 1.24 \\
Sex & 13.4 & 5.69 & 1.33 \\
Age & 17.6 & 0.21 & 0.06 \\
Hypercholesterolaemia & 22.3 & 4.31 & 1.29 \\
Diabetes & 24.7 & 4.31 & 1.69 \\
\hline
\end{tabular}

MI, myocardial infarction. was incomplete the original angiograms were reviewed. All angiograms were reviewed by a single observer.

\section{IDENTIFYING BETTER PREDICTORS OF}

ANGIOGRAPHIC FINDINGS

In 178 consecutive patients undergoing angiography (the 125 patients described above, plus an additional 53 patients), clinical data were collected as described above, and additional information was obtained concerning previous infarction, diabetes, hypercholesterolaemia, and hypertension (table 3). Diabetes was defined as a recorded diagnosis of diabetes or the prescription of antidiabetic drugs. Hypercholesterolaemia was defined as a plasma cholesterol concentration of greater than $6.5 \mathrm{mmol} / \mathrm{l}$. Hypertension was defined as a recorded diagnosis of hypertension or a systolic blood pressure of greater than $160 \mathrm{~mm} \mathrm{Hg}$ or a diastolic blood pressure of greater than $100 \mathrm{~mm} \mathrm{Hg}$. Angiograms were scored as described above. Univariate and multivariate regression analysis were used to identify predictors of the angiographic score. Variables tested were age in years, diabetes, hypertension, hypercholesterolaemia, previous myocardial infarction, previous cardiac surgery, unstable angina, number of antianginal drugs, dyspnoea, sex, and smoking.

For multivariate regression analysis variables were initially entered in order of strength of correlation on univariate analysis.

MEASURING THE PERFORMANCE OF A MODIFIED SCORING SYSTEM

The modified scoring system incorporating changes resulting from multivariate analysis was compared to the original system in a new set of 100 consecutive patients.

DATA HANDLING AND STATISTICAL ANALYSIS

Datasets were maintained in DBase IV and Microsoft Excel. Statistical analysis used SPSS for Windows.

\section{Results}

INITIAL MAPPING OF WAITING LIST PATIENTS TO A CLINICAL SCORING SYSTEM

Outpatient booking forms were returned for 1150 patients, of whom 665 had a primary diagnosis of ischaemic heart disease and were scheduled for diagnostic coronary angiography. All these patients were scored according to the system shown in table 1 . Patients excluded were 263 scheduled for PTCA after previous angiography, 122 for electrophysiology or cardioversion, and 100 with valve disease.

The cumulative distribution of the scores for the 665 patients with a primary diagnosis of ischaemic heart disease is shown in fig 1 . For any given score on the $\mathrm{X}$ axis, this graph indicates on the $\mathrm{Y}$ axis the percentage of patients with at least that score.

By September 1997, 572 patients had undergone angiography, seven had died, and 83 were still awaiting angiography or had transferred to the private sector. Data on three patients were missing. Figure 2 shows the relation between score and postangiography management decision. 
Table 5 Scoring factors in non-invasive prioritisation scheme: modified version

\begin{tabular}{llr}
\hline Heading & Details & Score \\
\hline Angina & Class I: angina on strenuous exertion & 1 \\
& Class II: angina on walking/climbing stairs rapidly & 2 \\
& Class III: angina walking one or two level blocks & 8 \\
Exercise test & Class IV: unstable angina/rest pain & 18 \\
& Bruce stage IV or negative at stage III & 0 \\
& Stage III positive & 8 \\
& Stage II positive & 12 \\
Age (years) & Stage I positive & 22 \\
Sex & Could not exercise & 10 \\
Diabetes & >9 64 & 4 \\
High cholesterol & Male & 8 \\
Previous myocardial infarction & 10 \\
& & 7 \\
\hline
\end{tabular}

COMPARISON OF CLINICAL AND ANGIOGRAPHIC SCORES

Comparison of the original clinical and angiographic scores in 125 consecutive patients who had undergone coronary angiography showed a poor correlation $(r=0.05)$. Bland-Altman analysis (results not shown) did not suggest any trend to better or worse correlation with the magnitude of the score.

IDENTIFYING BETTER PREDICTORS OF

ANGIOGRAPHIC FINDINGS

Because of the limited ability of symptom and exercise test related variables to predict the degree of coronary obstruction found at angiography, we used regression analysis to investigate the predictive value of a series of additional variables.

Significant associations on univariate analysis were found between coronary score and age, diabetes, previous myocardial infarction, hypercholesterolaemia, previous cardiac surgery,

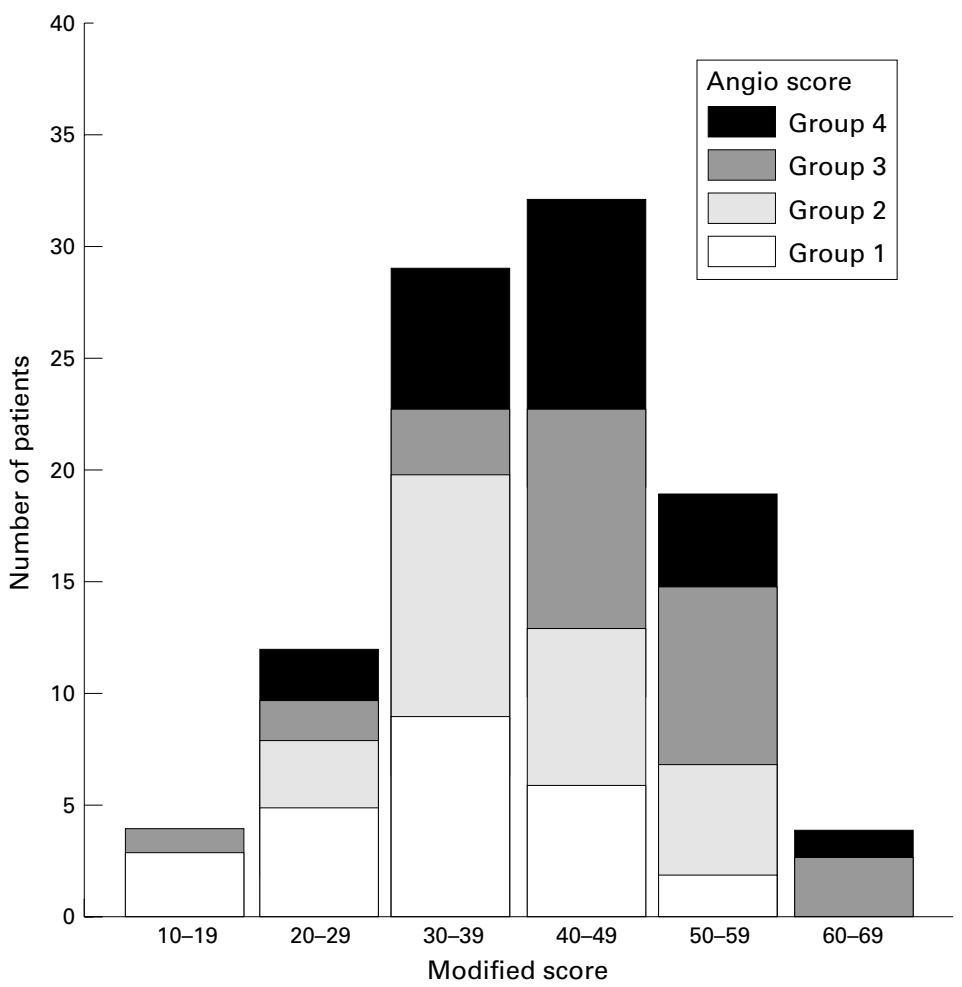

Figure 3 Ability of the modified scoring system to predict patients' angiographic scores (grouped as described in the text) in a new test group of 100 consecutive patients.

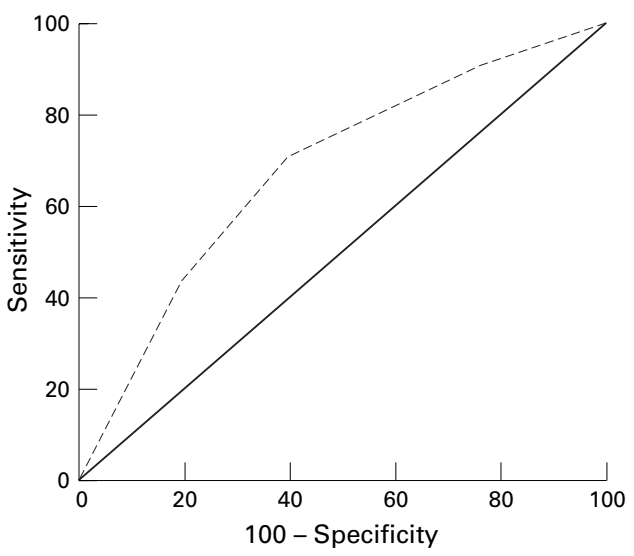

Figure 4 Receiver operating characteristic curve (\% sensitivity $v 100-\%$ specificity) for the ability of the modified scoring system to identify patients with angiographic scores in the two highest categories (groups 3 or 4) in a new test group of 100 patients.

number of antianginal drugs, and sex. The five variables that were independent predictors of coronary score on multivariate analysis were sex, previous myocardial infarction, age, diabetes, and hypercholesterolaemia. The cumulative $R^{2}$ values and coefficients for these variables are given in table 4 . The scoring system was modified to include these variables, with weightings determined by the regression coefficients (table 5).

\section{MEASURING THE PERFORMANCE OF A MODIFIED} SCORING SYSTEM

The group used to derive the modified scoring system and the group used to test it are compared in table 3 . The groups were similar apart from a higher incidence of previous myocardial infarction and more patients on multiple antianginal drugs in the index group. The ability of the modified scoring system to predict patients' angiographic scores in a new test group of 100 patients is shown in fig 3 .

Because the angiographic score (table 2) is not truly continuous, patients were divided into the following four groups, which approximate to quartiles: group 1 (score < 14), $\mathrm{n}=25$, patients with normal coronaries or mild coronary disease; group 2 (score 15-18), $\mathrm{n}=$ 26 , moderate disease-for example, two vessel disease $(>90 \%)$ not involving left anterior descending coronary artery (LAD); group 3 (score 19-26), $\mathrm{n}=27$, more severe disease-for example, three vessel disease with $75-89 \%$ stenosis involving proximal LAD; group 4 (score $27-36), \mathrm{n}=22$, very severe coronary disease including left main coronary stenosis. Patients in groups 3 and 4 have patterns of coronary disease for which it is widely accepted that intervention is indicated on prognostic grounds. For identifying patients with a coronary score of 19 or higher, a score of 20 had a sensitivity of $98 \%$ and a specificity of $6 \%$, a score of 30 had a sensitivity of $89 \%$ and a specificity of $22 \%$, and a score of 40 had a sensitivity of $71 \%$ and a specificity of $60 \%$. Figure 4 shows a receiver operating characteristic curve for the ability of the modified scoring system to identify patients with angiographic scores in groups 3 or 4 . Figure 5 shows the 


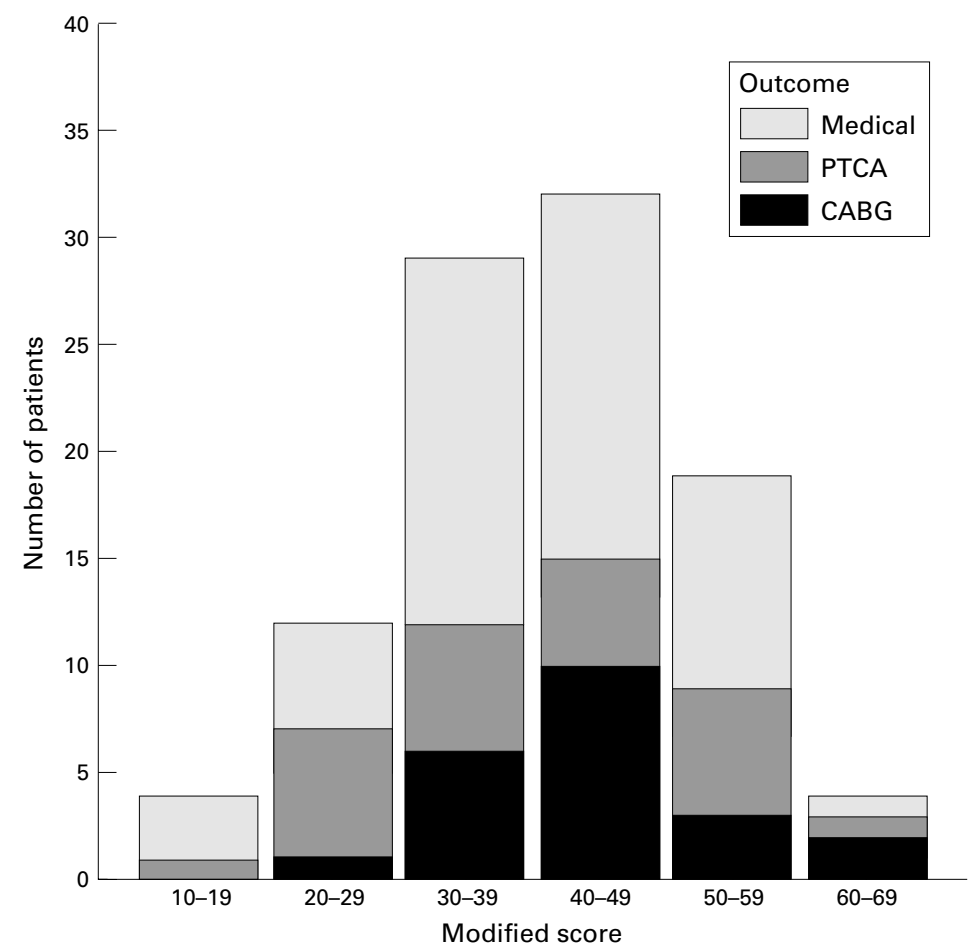

Figure 5 Relation between modified score and clinical outcome in a new test group of 100 patients. retrospectively, both in patients undergoing angiography and in patients being considered for intervention. ${ }^{9-13}$ These studies have given high appropriateness scores to severely symptomatic patients with objective restriction of exercise tolerance and persisting symptoms despite drug treatment, particularly when combined with extensive coronary disease.

Our scoring system is successful in giving a relatively low priority to patients who subsequently had relatively minor coronary disease at angiography, but also underestimated the severity of coronary disease in a small but significant proportion of patients. Is an imperfect prioritisation system better or worse than no prioritisation system at all? The answer will depend on the prevalence of coronary disease in the population studied, on the ultimate objectives of prioritisation, and on the stringency with which the prioritisation system has to be applied. Decisions on appropriateness cannot be made in isolation from financial considerations. In the present study we have not sought to define appropriateness, but to investigate whether non-invasive data could be used for prioritising patients as a step towards both fairness in the allocation of resources and a clearer understanding of what a given level of funding will and will not purchase.

relation between score and clinical outcome. A score of 40 had a sensitivity of $67 \%$ and a specificity of $50 \%$ for predicting coronary artery bypass graft surgery (CABG), a sensitivity of $47 \%$ and a specificity of $40 \%$ for percutaneous transluminal coronary angioplasty (PTCA).

\section{Discussion}

The data show that it is possible to carry out a ranking exercise based on clinical data collected out the time of entry to the catheter waiting list. We confirmed previous work showing a poor correlation between symptom severity or exercise testing and coronary anatomy, although both are valid independent predictors of outcome. ${ }^{8}{ }^{9}$ The modified scoring system does have some ability to predict severity of coronary disease, as well as reflecting symptom severity and exercise tolerance. It is possible that prediction could be improved by using cholesterol as a continuous variable, and by including other variables such as lipoprotein (a), but at the cost of complexity. Using a larger series for the multivariate analysis might have identified more predictors, but their additional contribution would be small. Both the original and the modified clinical scores were only modestly successful in predicting clinical outcome, but they were being tested in a selected population that already had a high probability of going on to PTCA or CABG. So far as we are aware there has been no previous systematic attempt to apply specific numerical scores prospectively to defined clinical features of patients being considered for coronary angiography, and to base selection on the result, although the concept was suggested by Gray and Hampton. ${ }^{7}$ There have, however, been several attempts to evaluate "appropriateness"

\section{PRACTICAL IMPLEMENTATION OF THE}

PRIORITISATION SYSTEM

Since the beginning of financial year 1997, circumstances have compelled us to implement a rationing system based on the work described above for patients who are the responsibility of our major purchaser. A simple proforma has been designed, which is completed in the clinic at the time the patient is considered for angiography. Although we have not specifically validated its use, practical considerations have led us to include myocardial perfusion scanning as an alternative to exercise electrocardiography for those unable to exercise, and for women. ${ }^{14}$

We knew at the beginning of the financial year the total number of cases the purchaser would fund. Allowance was made for emergency admissions (estimated from previous experience) and for patients already on the waiting list. Reference to the cumulative frequency curve for the previous year (fig 1) suggested a threshold value that patients needed to achieve before they were placed on the waiting list. For the current financial year, in respect of our principal purchaser this was 40 points. Patients who do not reach the threshold value are being told that they have not reached the level of priority specified by their purchaser. Some of these patients may wish to discuss alternative ways of funding their investigation. Any patient considered for angiography who does not reach the specified threshold is nevertheless recorded and followed up. If symptoms or circumstances change the scoring process is repeated. The threshold value is reviewed monthly in the light of progress with the contract, number of emergency admissions, and any additional sources of funding. We are naturally concerned that even at this threshold we are excluding a 
proportion of patients with severe coronary disease and this will be an increasing problem if the threshold should rise higher. In fact, the trend over the past six months has been for a progressive shortening of our waiting list, and by the end of next year it should be down to three months. This, together with a promise of additional purchasing, should enable us cautiously to lower our threshold.

1 Task Force of the European Society of Cardiology. Management of stable angina pectoris. Eur Heart 7 1997;18:394ment

2 Black N, Langham S, Coshall C, et al. Impact of the 1991 NHS reforms on the availability and use of coronary revascularisation in the UK 1987-1995. Heart 1996;76(suppl 4):1-25

3 Clinical Standards Advisory Group. Coronary artery bypass grafting and coronary angioplasty. Access to and availability of specialist services. Report of a CSAG working group. London: HMSO, 1993.

4 King's Fund Consensus Development Conference Panel. Coronary artery bypass grafting. BMF 1984;289:1527-9.

5 Joint Cardiology Committee of the RCP (London) and RCS (England). Provision of services for the diagnosis and treatment of heart disease. Br Heart f 1992;67:106-16.
6 de Bono DP, Hopkins A, for a joint working group of the British Cardiac Society and RCP (London). The management of stable angina: guidelines and audit standards. $\mathcal{F} R$ Coll Physicians (Lond) 1993;27:267-73.

7 Gray D, Hampton JR. Methods of establishing criteria for purchasing coronary angiography in the investigation of chest pain. F Public Health 1994;16:399-405.

8 Hadorn DC, Holmes AC. The New Zealand priority criteria project-part 2: coronary artery bypass graft surgery. BMF 1997;314:135-8.

9 Mark DB, Nelson CL, Califf RM, et al. Continuing evolution of therapy for coronary artery disease. Circulation 1994;89:2015-25.

10 Noonan SJ, Cook JL, Likeller CE, et al. Relationship of anatomic disease to appropriateness ratings of coronary angiography. Arch Intern Med 1995;155:1209-13.

11 Cleland JGF, Walker A. Is medical treatment for angina the most cost effective option? Eur Heart $\mathcal{F}$ 1997;18(suppl B):B35-40.

12 Gray D, Hampton JR. Variations in the use of coronary angiography in three cities in the Trent Region. Br Heart 7 1994;71:474-8.

13 Kadel C, Burger W, Klepzig H. Qualitätssicherung in der invasiven Kardiologie: eine prospektive Untersuchung zurBewertung von Indkationen zur Koronarangiographie und zur Koronardilatation nach der methode der "RAND Corporation." Dtsch Med Wochenschr 1996;121:465-71.

14 Curzen N, Patel D, Clarke D, et al. Women with chest pain: is exercise testing worthwhile? Heart 1996;76:156-60. 